Algebra Univers. 69 (2013) 231-235

DOI $10.1007 / \mathrm{s} 00012-013-0230-7$

Published online March 30, 2013

(c) 2013 The Author(s)

Algebra Universalis

This article is published with open access at Springerlink.com

\title{
Defining subdirect product closed classes in infinitary logic
}

\author{
Michą M. Stronkowski
}

Abstract. We note that a class of models is subdirect product closed if and only if it is definable by a class of $L_{\infty \infty}$-sentences of a special form.

\section{Introduction}

By a weak implication we mean a sentence in $L_{\infty \infty}$ of the form

$$
(\forall \bar{x})(\exists y)\left[[y \in \bar{x}] \wedge \bigwedge_{i \in I} \varrho_{i}(\bar{x}) \rightarrow \delta(\bar{x})\right],
$$

where $\varrho_{i}$ for $i \in I$, and $\delta$ are atomic first-order formulas, $\bar{x}=\left(x_{k}\right)_{k \in K}$, and $[y \in \bar{x}]$ is an abbreviation of $\bigvee_{k \in K}\left[y=x_{k}\right]$. Note that $(\omega)$ is equivalent to

$$
(\forall \bar{x})\left[\left[(\forall y)[y \in \bar{x}] \wedge \bigwedge_{i \in I} \varrho_{i}(\bar{x})\right] \rightarrow \delta(\bar{x})\right]
$$

and $\mathbf{M} \models(\forall y)[y \in \bar{a}]$ if and only if the valuation $x_{i} \mapsto a_{i}$ is onto $M$. Thus, $\mathbf{M}$ satisfies $(\omega)$ if and only if $\mathbf{M} \models \bigwedge_{i \in I} \varrho_{i}(\bar{a})$ yields $\mathbf{M} \models \delta(\bar{a})$, for every valuation $x_{i} \mapsto a_{i}$ onto $M$.

A model $\mathbf{M}$ is a subdirect product of models $\mathbf{M}_{s}, s \in S$, if $\mathbf{M}$ is a submodel of the direct product $\prod_{s \in S} \mathbf{M}_{s}$ and all projections of the product restricted to $\mathbf{M}$ are onto [2]. We write then $\mathbf{M} \leqslant_{S D} \prod_{s \in S} \mathbf{M}_{s}$. A class $\mathcal{C}$ of models is subdirect product closed if every subdirect product of models from $\mathcal{C}$ also belongs to $\mathcal{C}$. (All considered classes are assumed to be classes of models in the same fixed first-order language $L$ and are closed under taking isomorphic images.)

In this note we would like to present the following observation.

Theorem. A class is subdirect product closed if and only if it is definable by a class of weak implications.

The proof of this fact is obtained by appropriate modifications in the proof of A. Shafaat's theorem [11], which states that the class of models is submodel

Presented by J. Adamek.

Received December 29, 2011; accepted in final form July 9, 2012.

2010 Mathematics Subject Classification: Primary: 08B26; Secondary: 03C75, 08C10.

Key words and phrases: subdirect product closed classes, weak implications, infinitary logic, axiomatizations.

This work was supported by Polish National Science Centre grant number DEC2011/01/D/ST1/06136. 
and direct product closed (is a prevariety) if and only if it is definable by possibly infinite implications.

In Section 3, we describe an example showing that we cannot obtain an axiomatization by a set of infinite sentences of a bit more general form than weak implications even for the class $\mathrm{P}_{\mathrm{S}}(\mathbf{M})$ of all subdirect products of a given finite model $\mathbf{M}$. This is contrary to the situation for prevarieties.

Classes typically considered in universal algebra, such as prevarieties, quasivarieties, and varieties, are subdirect product closed. But the fact that they are closed under additional operations, such as taking subalgebras, ultraproducts, and homomorphic images, makes their axiomatizations simpler. Classes of models closed solely under taking subdirect products appear in abstract algebraic logic as classes of Suszko reduced matrices for deductive systems [5], in particular as classes of reduced matrices for protoalgebraic deductive systems [4]. In the protoalgebraic case though, we also have a simpler axiomatization. Indeed, by [3, Theorem 13.10], such classes may be defined by finite implications (quasi-identities) and one possibly infinite sentence of the form $(\forall x, y)(\exists \bar{z})\left[\bigwedge_{i \in I} \varrho_{i}(x, y, \bar{z}) \rightarrow x \approx y\right]$, where $\varrho_{i}$ are atomic first-order formulas and there are only countable many variables in $\bar{z}$. However, no particular axiomatization is known in the general case.

The problem of the existence of a first-order axiomatization for $\mathrm{P}_{\mathrm{S}}(\mathbf{M})$, where $\mathbf{M}$ is finite, was considered by $\mathbf{G}$. Grätzer in [7], and later revisited by J. T. Baldwin and M. A. Samhan in [1], where certain sufficient conditions were presented.

Let us recall an important result of $\mathrm{R}$. C. Lyndon $[10,9]$. He showed that subdirect product closed classes that are first-order definable are in fact definable by first-order special Horn sentences.

Finally, note that there is an easy semantical characterization of subdirect product closed classes. For a class $\mathcal{C}$ and a model $\mathbf{M}$ let $\operatorname{Con}_{\mathcal{C}}(\mathbf{M})$ be the ordered set of congruences $\alpha$ of $\mathbf{M}$ such that $\mathbf{M} / \alpha \in \mathcal{C}$. For algebras the order is set inclusion, and for models it is pairwise set inclusion (see [6] for the definition of congruence for models). Then $\mathcal{C}$ is subdirect product closed if and only if $\operatorname{Con}_{\mathcal{C}}(\mathbf{M})$ is a complete lattice, for every $\mathbf{M}$.

\section{Proof of the theorem}

We start by verifying the easier forward direction of the Theorem.

Lemma 2.1. The subdirect product operation preserves the satisfaction of weak implications.

Proof. Let $\sigma$ be a weak implication of the form $(\omega)$, let $\mathbf{M}_{s}, s \in S$, be models satisfying $\sigma$, let $\mathbf{M} \leqslant S D \prod_{s \in S} \mathbf{M}_{s}$ be a subdirect product, and let $x_{i} \rightarrow a_{i}=$ $\left(a_{i}^{s}\right)_{s \in S}$ be a valuation onto $M$ such that $\mathbf{M} \models \bigwedge_{i \in I} \varrho_{i}(\bar{a})$. Then

$$
\mathbf{M}_{s}=\left[\bigwedge_{i \in I} \varrho_{i}\left(\bar{a}^{s}\right) \rightarrow \varrho\left(\bar{a}^{s}\right)\right] \wedge\left[\bigwedge_{i \in I} \varrho_{i}\left(\bar{a}^{s}\right)\right]
$$


and hence $\mathbf{M}_{s}=\delta\left(\bar{a}^{s}\right)$. This proves that $\mathbf{M}=\delta(\bar{a})$.

Let us now fix a subdirect product closed class $\mathcal{C}$. For every model $\mathbf{M}$ (not necessarily in $\mathcal{C}$ ) define

$$
\Phi_{\mathbf{M}}=\left\{\varphi: \mathbf{M} \rightarrow \mathbf{M}_{\varphi} \mid \varphi \text { is onto, } M_{\varphi} \subseteq M, \text { and } \mathbf{M}_{\varphi} \in \mathcal{C}\right\} .
$$

Let $\eta_{\mathbf{M}}: \mathbf{M} \rightarrow \prod_{\varphi \in \Phi_{\mathbf{M}}} \mathbf{M}_{\varphi}$ be the homomorphism given by $\eta_{\mathbf{M}}(a)(\varphi)=\varphi(a)$. Put $\mathrm{R}(\mathbf{M})=\eta_{\mathbf{M}}(\mathbf{M})$. Next let $\bar{x}=\left(x_{a}\right)_{a \in M}$ be a sequence of distinct variables and $x_{a} \mapsto a$ be the valuation onto $M$. Define $\Omega_{\mathbf{M}}$ to be the set of all weak implications of the form

$$
(\forall \bar{x})(\exists y)[\bigwedge\{\varrho(\bar{x}) \mid \varrho \text { is atomic, } \mathbf{M} \models \varrho(\bar{a})\} \wedge[y \in \bar{x}] \rightarrow \delta(\bar{x})],
$$

where $\mathrm{R}(\mathbf{M}) \models \delta\left(\eta_{\mathbf{M}}(\bar{a})\right)$. Note that $\mathbf{M} \models \Omega_{\mathbf{M}}$ yields $\mathbf{M} \cong \mathbf{R}(\mathbf{M})$. Finally, put $\Omega=\bigcup_{M} \Omega_{M}$.

Lemma 2.2. The class of weak implications $\Omega$ defines $\mathcal{C}$.

Proof. Assume that $\mathbf{M} \models \Omega$. Then in particular, $\mathbf{M} \models \Omega_{\mathbf{M}}$, and we have $\mathbf{M} \cong \mathrm{R}(\mathbf{M}) \leqslant S D \prod_{\varphi \in \Phi_{\mathbf{M}}} \mathbf{M}_{\varphi}$. Hence, $\mathbf{M} \in \mathcal{C}$.

Now suppose that $\mathbf{M} \in \mathcal{C}$. Consider a model $\mathbf{N}$. Let $\bar{x}=\left(x_{b}\right)_{b \in N}$ be a sequence of distinct variables and $x_{b} \mapsto a_{b}$ a valuation onto $M$. Assume that $\mathbf{M} \models \varrho(\bar{a})$ whenever $\mathbf{N} \models \varrho(\bar{b})$ for every atomic $\varrho(\bar{x})$ (here we employ the valuation $\left.x_{b} \mapsto b\right)$. This means that the mapping $\varphi: b \mapsto a_{b}$ is a homomorphism from $\mathbf{N}$ onto $\mathbf{M}$. Without loss of generality, we may assume that $M \subseteq N$, and hence $\varphi \in \Phi_{\mathbf{N}}$. Let $\pi: \mathrm{R}(\mathbf{N}) \rightarrow \mathbf{M}$ be the restriction of the $\varphi$-th projection. Then $\pi \circ \eta_{\mathbf{N}}=\varphi$. This yields that whenever $\delta(\bar{x})$ is an atomic formula such that $\mathrm{R}(\mathbf{N}) \models \delta\left(\eta_{\mathbf{N}}(\bar{b})\right)$, then $\mathbf{M} \models \delta(\bar{a})$. This proves that $\mathbf{M} \models \Omega_{\mathbf{N}}$.

\section{Example}

If we can define a subdirect product closed class $\mathcal{C}$ by weak implications in which the number of universal quantifiers is bounded by a cardinal $\kappa$, then all models of cardinality greater than $\kappa$ belong to $\mathcal{C}$. Thus, we cannot expect in general an axiomatization by a set of weak implications. The following example shows that it is also the case for finitely generated subdirect product closed classes even if we allow sentences of a bit more general form than weak implications.

Let $\forall^{\kappa} \exists^{\infty}-L_{\infty \infty}$ be the language consisting of formulas in $L_{\infty \infty}$ of the form $(\forall \bar{x})(\exists \bar{y}) \gamma(\bar{x}, \bar{y})$, where $\bar{x}$ consists of less than $\kappa$ variables and $\gamma$ is quantifier free.

Lemma 3.1. Let $\sigma$ be a sentence in $\forall^{\kappa} \exists^{\infty}-L_{\infty \infty}$. If

$$
(\forall C \subseteq M)[|C| \leqslant \kappa \text { implies }(\exists \mathbf{N} \leqslant \mathbf{M})[C \subseteq N \text { and } \mathbf{N} \mid=\sigma]],
$$

then $\mathbf{M} \models \sigma$. 
Let $\mathbf{A}=(\{0,1,2\},+)$ be the algebra where the operation is given by the table

\begin{tabular}{c|ccc}
+ & 0 & 1 & 2 \\
\hline 0 & 0 & 0 & 0 \\
1 & 1 & 0 & 1 \\
2 & 2 & 2 & 0
\end{tabular}.

R. McKenzie showed that $\mathrm{P}_{\mathrm{S}}(\mathbf{A})$ is not definable in first-order logic [8, Chapter 7, Exercises 55-58]. We slightly modify his argument in order to obtain the following fact.

Fact 3.2. The class $\mathrm{P}_{\mathrm{S}}(\mathbf{A})$ is not definable in $\forall^{\kappa} \exists^{\infty}-L_{\infty}$ for any cardinal $\kappa$.

Proof. We may assume that $\kappa$ is infinite. Let $\lambda$ be a cardinal greater than $\kappa$. For $f \in A^{\lambda}$, define $\operatorname{supp}(f)=\{i \in \lambda \mid f(i) \neq 0\}$ and put

$$
B=\left\{f \in A^{\lambda} \mid f(0) \in\{0,1\} \text { and }|\operatorname{supp}(f)| \leqslant \kappa\right\} .
$$

Clearly, $B$ is a carrier of the subalgebra $\mathbf{B}$ of $\mathbf{A}^{\lambda}$. Note that for $f, g \in B$,

$$
\operatorname{supp}(f) \cap \operatorname{supp}(g)=\emptyset \Rightarrow f+g=f .
$$

Let us distinguish two elements $f_{0}, f_{1}$ in $B$ by $f_{0}(0)=0, f_{1}(0)=1$, and $f_{0}(i)=f_{1}(i)=0$ for $i \neq 0$. Then for every $f \in B$, we have $f+f=f_{0}$ and $f_{0}$ is the only idempotent element in $\mathbf{B}$.

We will obtain the aim by verifying that every sentence in $\forall^{\kappa} \exists^{\infty}-L_{\infty}$ valid in $\mathrm{P}_{\mathrm{S}}(\mathbf{A})$ is also valid in $\mathbf{B}$ and showing that $\mathbf{B} \notin \mathrm{P}_{\mathrm{S}}(\mathbf{A})$. The first fact follows from the following claim and Lemma 3.1.

Claim. For every subset $C$ of $B$ with $|C| \leqslant \kappa$, there exists $\mathbf{D} \leqslant \mathbf{B}$ such that $C \subseteq D$ and $\mathbf{D} \in \mathrm{P}_{\mathrm{S}}(\mathbf{A})$.

Let $I=\bigcup_{f \in C} \operatorname{supp}(f)$. As $|C| \leqslant \kappa$, so $|I| \leqslant \kappa$ and there exists $i^{*} \in \lambda-I$. Let $h$ be the element of $B$ such that $h\left(i^{*}\right)=1$ and $h(i)=0$ for $i \neq i^{*}$. Put

$$
E=\{f \in B \mid \operatorname{supp} f \subseteq I\}
$$

and $D=E \cup\{h\}$. Clearly, $\mathbf{E} \leqslant \mathbf{B}$, and by (L0), $\mathbf{D} \leqslant \mathbf{B}$. Observe that for a homomorphism $\psi: \mathbf{E} \rightarrow \mathbf{A}$ and an element $a \in A-\psi(E)$, the mapping given by $\varphi(g)=\psi(g)$ for $g \in E$ and $\varphi(h)=a$ is a homomorphism from $\mathbf{D}$ into $\mathbf{A}$. In order to show that $\mathbf{D} \in \mathrm{P}_{\mathrm{S}}(\mathbf{A})$, we have to find an onto homomorphism $\varphi: \mathbf{D} \rightarrow \mathbf{A}$ separating $g_{1}$ and $g_{2}$ for every pair $g_{1}, g_{2}$ of distinct elements in $D$. If $g_{1}(i) \neq g_{2}(i)$ for some $i \in I-\{0\}$, then as $\varphi$ we can take the projection on the $i$-th coordinate. If $g_{1}(0) \neq g_{2}(0)$ or $\left\{g_{1}, g_{2}\right\}=\left\{f_{0}, h\right\}$, let $\psi: \mathbf{E} \rightarrow \mathbf{A}$ be the projection on the 0 -th coordinate and define $\varphi(g)=\psi(g)$ for $g \in E$ and $\varphi(h)=2$.

Claim. $\mathbf{B} \notin \mathrm{P}_{\mathrm{S}}(\mathbf{A})$.

Let $\varphi: \mathbf{B} \rightarrow \mathbf{A}$ be any homomorphism separating $f_{0}$ and $f_{1}$. The element $f_{0}$, as the only idempotent one in $\mathbf{B}$, must be mapped by $\varphi$ onto 0 . Assume that $\varphi\left(f_{1}\right)=a \neq 0$. For $f \in B$ satisfying $f(0)=1$, the equality $f_{1}+f=f_{0}$ 
yields $\varphi(f)=a$. Now for $g \in B$ such that $g(0)=0$, let $g^{\prime}(0)=1$ and $g^{\prime}(i)=g(i)$ for $i \neq 0$. Since $\kappa$ is infinite, $g^{\prime} \in B$. Then $\varphi\left(g^{\prime}\right)=a, g+g^{\prime}=f_{0}$, and hence $\varphi(g) \in\{0, a\}$. Thus, $\varphi$ is not onto.

Note however that for a finite model $\mathbf{M}$ in a finite language, the class $\mathrm{P}_{\mathrm{S}}(\mathbf{M})$ is definable in $L_{\aleph_{1} \aleph_{1}}$ by a sentence of the form $(\forall \bar{x})(\exists \bar{y})(\forall \bar{z}) \gamma(\bar{x}, \bar{y}, \bar{z})$, where $\gamma$ is quantifier free and $\bar{x}, \bar{y}$ are finite [7, Point 3.3].

\section{REFERENCES}

[1] Baldwin, J.T., Samhan, M.A.: On the definability of $P_{S}(A)$. Algebra Universalis 23(3), 254-261 (1986). DOI 10.1007/BF01230620. URL http://dx.doi.org/10.1007/BF01230620

[2] Birkhoff, G.: Subdirect unions in universal algebra. Bull. Amer. Math. Soc. 50, 764-768 (1944)

[3] Blok, W.J., Pigozzi, D.: Algebraic semantics for universal Horn logic without equality. In: Universal algebra and quasigroup theory (Jadwisin, 1989), Res. Exp. Math., vol. 19, pp. 1-56. Heldermann, Berlin (1992)

[4] Czelakowski, J.: Protoalgebraic logics, Trends in Logic-Studia Logica Library, vol. 10. Kluwer, Dordrecht (2001)

[5] Czelakowski, J.: The Suszko operator. Part I. Studia Logica 54(1-2), 181-231 (2003)

[6] Gorbunov, V.A.: Algebraicheskaya Teoriya Kvazimnogoobrazij. Nauchnaya Kniga, Novosibirsk (1999). English transl. Algebraic Theory of Quasivarieties, Consultants Bureau, New York (1998)

[7] Grätzer, G.: On the class of subdirect powers of a finite algebra. Acta Sci. Math. (Szeged) 25, 160-168 (1964)

[8] Grätzer, G.: Universal algebra, 2nd edn. Springer, New York (1979)

[9] Lyndon, R.C.: Properties preserved in subdirect products. Pacific J. Math. 9, 155-164 (1959)

[10] Lyndon, R.C.: Properties preserved under algebraic constructions. Bull. Amer. Math. Soc. 65, 287-299 (1959)

[11] Shafaat, A.: On implicationally defined classes of algebras. J. London Math. Soc. 44, 137-140 (1969)

\section{Michą M. Stronkowski}

Faculty of Mathematics and Information Sciences, Warsaw University of Technology, ul. Koszykowa 75, 00-662 Warsaw, Poland e-mail: m.stronkowski@mini.pw.edu.pl

Open Access This article is distributed under the terms of the Creative Commons Attribution License which permits any use, distribution, and reproduction in any medium, provided the original author(s) and the source are credited. 